OPEN ACCESS

Edited by:

Kai-Da Xu,

Xi'an Jiaotong University, China

Reviewed by:

Cheng Zhang,

Wuhan University of Technology,

China

Jia-Yuan Yin,

Xidian University, China

*Correspondence:

Guo Qing Luo

luoguoqing@hdu.edu.cn

Specialty section:

This article was submitted to

Optics and Photonics,

a section of the journal

Frontiers in Physics

Received: 09 January 2022 Accepted: 24 January 2022

Published: 17 February 2022

Citation:

Pan BC, Yu P, Guo BJ, Qian YH and Luo GQ (2022) Unequal Bandpass Filtering Power Divider Based on Hybrid HMSIW-SSPP Modes.

Front. Phys. 10:851150.

doi: 10.3389/fphy.2022.851150

\section{Unequal Bandpass Filtering Power Divider Based on Hybrid HMSIW-SSPP Modes}

\author{
Bai Cao Pan ${ }^{1,2}$, Ping Yu ${ }^{1}$, Ben Jian Guo ${ }^{1}$, Ya Hui Qian ${ }^{1}$ and Guo Qing Luo ${ }^{1 *}$ \\ ${ }^{1}$ Key Laboratory of RF Circuits and System Ministry of Education, School of Electronics and Information, Hangzhou Dianzi \\ University, Hangzhou, China, ${ }^{2}$ State Key Laboratory of Millimeter Waves, Southeast University, Nanjing, China
}

This letter presents a novel unequal bandpass filtering power divider based on hybridmode of half mode substrate integrated waveguide (HMSIW) and spoof surface plasmon polaritons (SSPPS). Bandpass response is achieved by combining the transmission properties of HMSIW and SSPPs simultaneously. The operating bandwidth can be designed in a wide range by simply optimizing the dimensions of HMSIW and SSPPs. In addition, defected ground structures (DGSs) are etched on the bottom of the substrate to improve out-of-band suppression. The power division ratio of the proposed unequal power divider is finally optimized to 1:3. The measured results agree with the simulated one. Such design provides a stable power division within wide frequency range from 6.5 to $9.5 \mathrm{GHz}$.

Keywords: unequal power divider, bandpass filtering, SSPPs, HMSIW, wide band

\section{INTRODUCTION}

The rapid development of wireless communication systems proposes more and more requirements for functional devices. In order to meet the market demands, different kinds of well-performed functional devices have been widely studied. In the past decade, substrate integrated waveguide (SIW) has become one of the hotspots in researches [1]. Considering its perfect high-pass transmission with low-loss, high-efficient properties, functional devices such as filter [2], power divider [3-6] and coupler [7] have been proposed. Half-mode SIW (HMSIW) consists of only half of SIW structures, while keeping the same performance as SIW $[8,9]$. Miniaturization can be realized. Spoof surface plasmon polaritons (SSPPs) are guiding surface modes along periodic metamaterial structures. Such modes have also received wide attention for its unique properties of perfect low-pass transmission and near-field confinement $[10,11]$. SSPPs have been studies in field of different highefficient designs such as bandpass filter [12, 13], power divider [14, 15], coupler [16], radiator [17].

Filters and power dividers are the most commonly-used functional devices in wireless communication systems. In certain applications, both kinds of devices are required to be integrated with each other to reduce the occupied space. And their performances are crucial for systems stability. In recent years, a series of filters and power dividers based SIW and SSPPs have been reported $[18,19]$. In order to improve the integration and operating properties, parasitic structures like resonators [20], interdigital structures (ISs) [21], defected ground structures (DGSs) $[22,23]$ have also been studied. The combination of SIW and SSPP provides a new solution for the miniaturization designs, since the power dividers of SIW are usually narrow-band and those of SSPP have limits of bulky dimensions.

In this letter, we proposed a novel unequal bandpass filtering power divider based on the hybrid HMSIW-SSPP modes, which shows compact size and power division ratio of 1:3. The hybrid unit can 


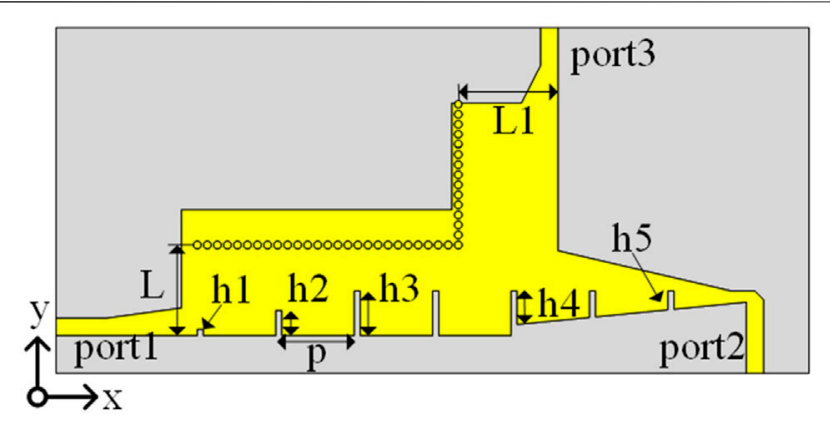

FIGURE 1 | Schematic of the proposed unequal filtering power divider.

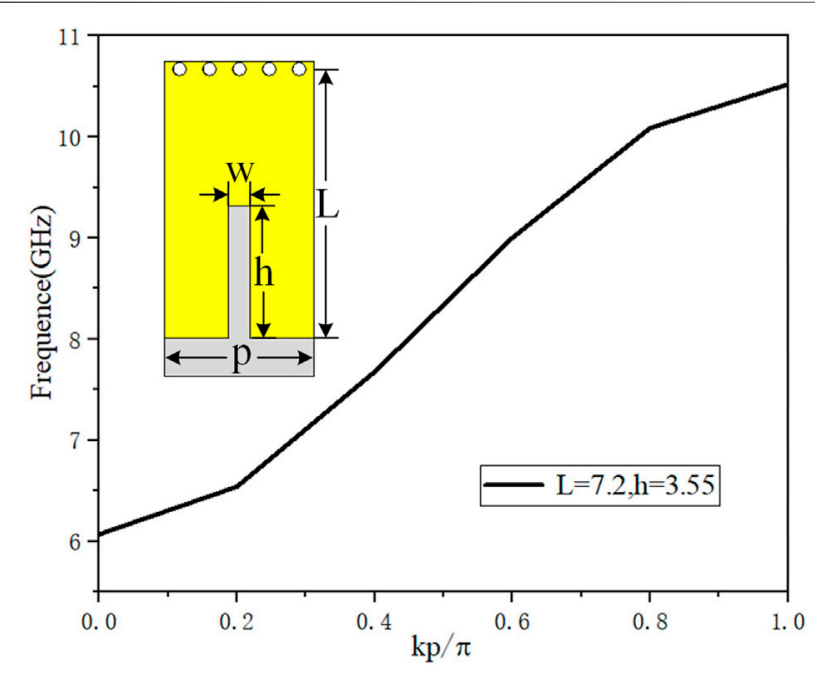

FIGURE 2 | The dispersion curves of the hybrid HMSIW-SSPP unit. Inset: the structure of the hybrid HMSIW-SSPP unit.

be compatible with the functional techniques of both HMSIW and SSPPs. The lower and upper edges of the passband can be controlled independently by changing the dimensions of HMSIW and SSPPs. Defected ground structure is also loaded aiming for improving out-of-band suppression.

\section{Design of Unequal Power Divider}

The schematic configuration of the proposed unequal filtering power divider is shown in Figure 1 with dimensions labelled. As it can be seen in the figure, periodic corrugated slots are etched on the top layer of HMSIW. Two slots with gradient groove depths of h1 and h2 are used to achieve broadband excitation of SSPP modes. Optimizing grooves' depths can help to improvement of the transmission efficiency. Two uniformed grooves of SSPP with depth of h3 are set as the transmission part. They collaborate with the HMSIW structures to provide the hybrid HMSIW-SSPP modes with unique transmission properties. The periodic interval of the SSPP units is p. At the output terminals, a SSPP channel of three unit matching structures with gradient grooves' depths and tapered edges are designed for momentum matching of SSPP at port 2. Meanwhile, another HMSIW channel is paralleled connected between the hybrid unit and the SSPP channel. The widths of both HMSIW structures are L and L1, respectively.

Figure 2 show the dispersion curves of the hybrid HMSIWSSPP unit. And its structure is shown in the inset of Figure 2. It can be observed from the figure that the cutoff frequency of HMSIW modes is lower than that of SSPP modes. Thus, bandpass response can be obtained. The hybrid unit provides bandpass property from 6.1 to $10.5 \mathrm{GHz}$. As is introduced in literatures, the cutoff frequencies of SIW and SSPP modes can be controlled by changing the width of SIW and the depth of SSPP grooves. For the proposed design, reduction of $\mathrm{L}$ or $\mathrm{h}$ leads to upper shifting of the lower or upper cutoff frequencies, respectively. Limited by the space allocation, SSPP grooves are always smaller than HMSIW. So the cutoff frequencies of SIW are always lower than those of SSPPs.

The dispersion properties of the hybrid HMSIW-SSPP unit under different parameters are compared in Figure 3. The unit with dimensions of $\mathrm{h}=3.5 \mathrm{~mm}, \mathrm{~L}=7 \mathrm{~mm}, \mathrm{w}=0.5 \mathrm{~mm}$ and $\mathrm{p}=$ $6 \mathrm{~mm}$ is used as reference and shown as the red dashed lines in the figure. Then each dimension is optimized and compared. In Figure 3A, the groove's depth in HMSIW unit is examined. When the depth h3 increases from 3 to $4 \mathrm{~mm}$, the upper cutoff frequencies shift from 11.2 to $9.4 \mathrm{GHz}$, while the lower cutoff frequencies keep at $6.1 \mathrm{GHz}$. Similarly, from Figure 3B it can be seen that when width L increases from 6 to $8 \mathrm{~mm}$, the lower cutoff frequencies of the hybrid unit reduce from $6.8 \mathrm{GH}$ to $5.5 \mathrm{GHz}$. Meanwhile, small range fluctuation between 10.1 and $10.3 \mathrm{GHz}$ of the upper cutoff frequencies is observed. The width of the groove $\mathrm{w}$ and its interval $\mathrm{p}$ can also be used for the modulation of operating band (shown in Figures 3C,D). The groove width $\mathrm{w}$ can change the upper cutoff frequencies in a small range. And the interval $p$ has a significant impact on the upper cutoff frequencies. Since the increasing interval would lead to excessive length of the device, depth $\mathrm{L}$ is usually used in modulation of operating band.

The simulated transmissions at port 3 of the unequal filtering power divider are illustrated in Figure 4 to testify the bandwidth modulation of the hybrid unit. As is compared in Figures 4A,B, upper cutoff frequencies or lower cutoff frequencies of $S_{31}$ shift to lower frequency band independently without apparently mutual influence. Based on the above analysis, a high-efficient passband from 6.5 to $9.5 \mathrm{GHz}$ of unequal filtering power divider can be designed. The simulated $S$ parameters are shown in Figure 5. In the whole passband, the reflection coefficient $S_{11}$ keeps below $-10 \mathrm{~dB}$, while the transmission coefficient $S_{21}$ and $S_{31}$ are around $-2.2 \mathrm{~dB}$ and $-7 \mathrm{~dB}$.

\section{OPTIMIZATION AND MEASUREMENT}

The DGS can disturb the current distribution on the metallic ground and introduce an extra transmission zero. In the optimized model, a two-element array of inverted T-shaped 

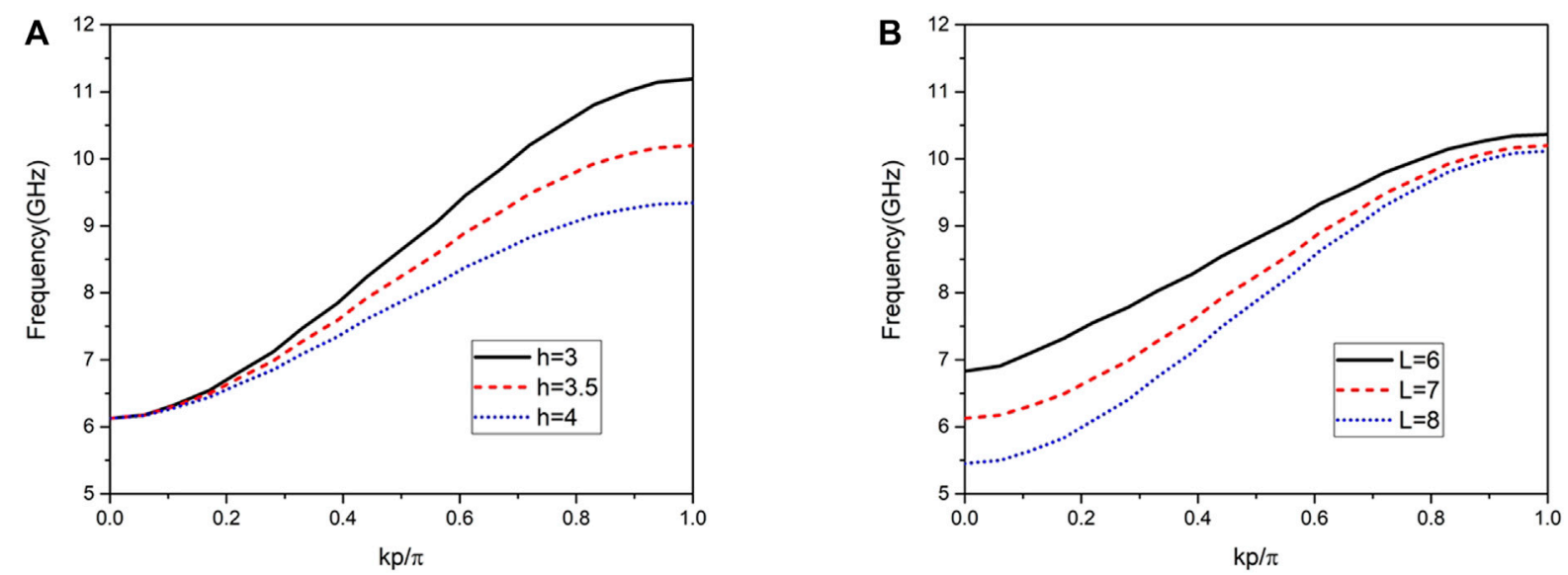

C

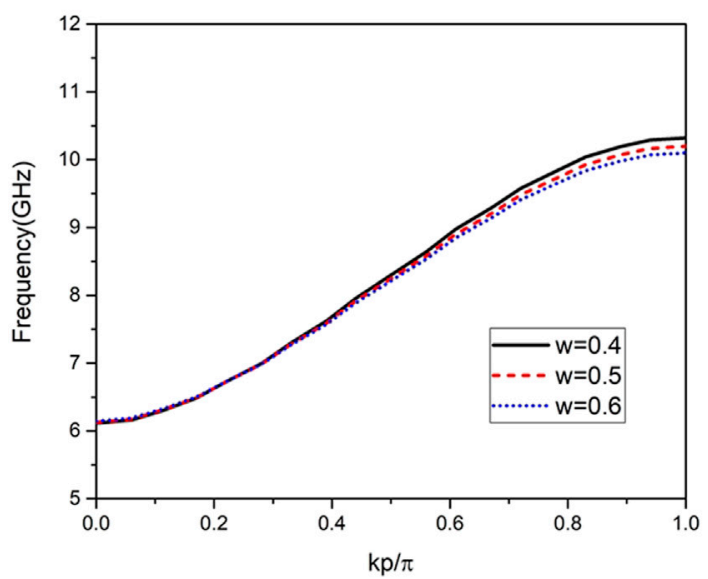

D

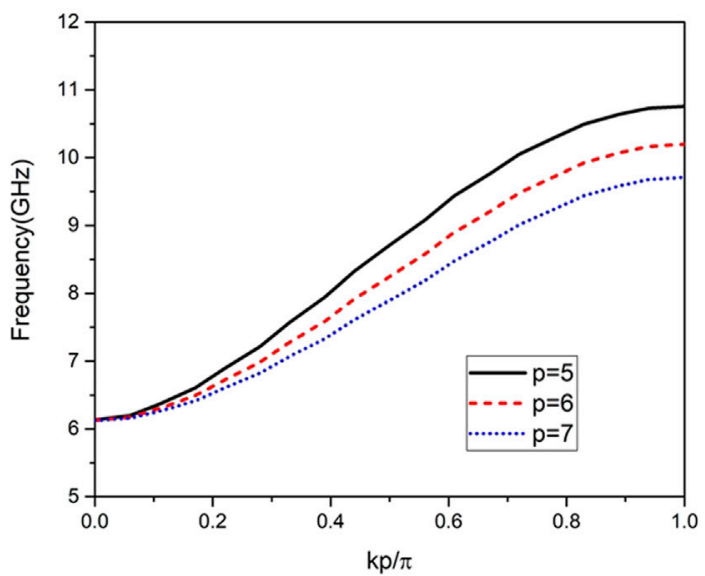

FIGURE 3 | The dispersion properties of the hybrid unit under different parameters of (A) depth of SSPP grooves h, (B) width of HMSIW L, (C) width of SSPP grooves $\mathrm{w}$, and (D) interval of SSPPs $\mathrm{p}$.
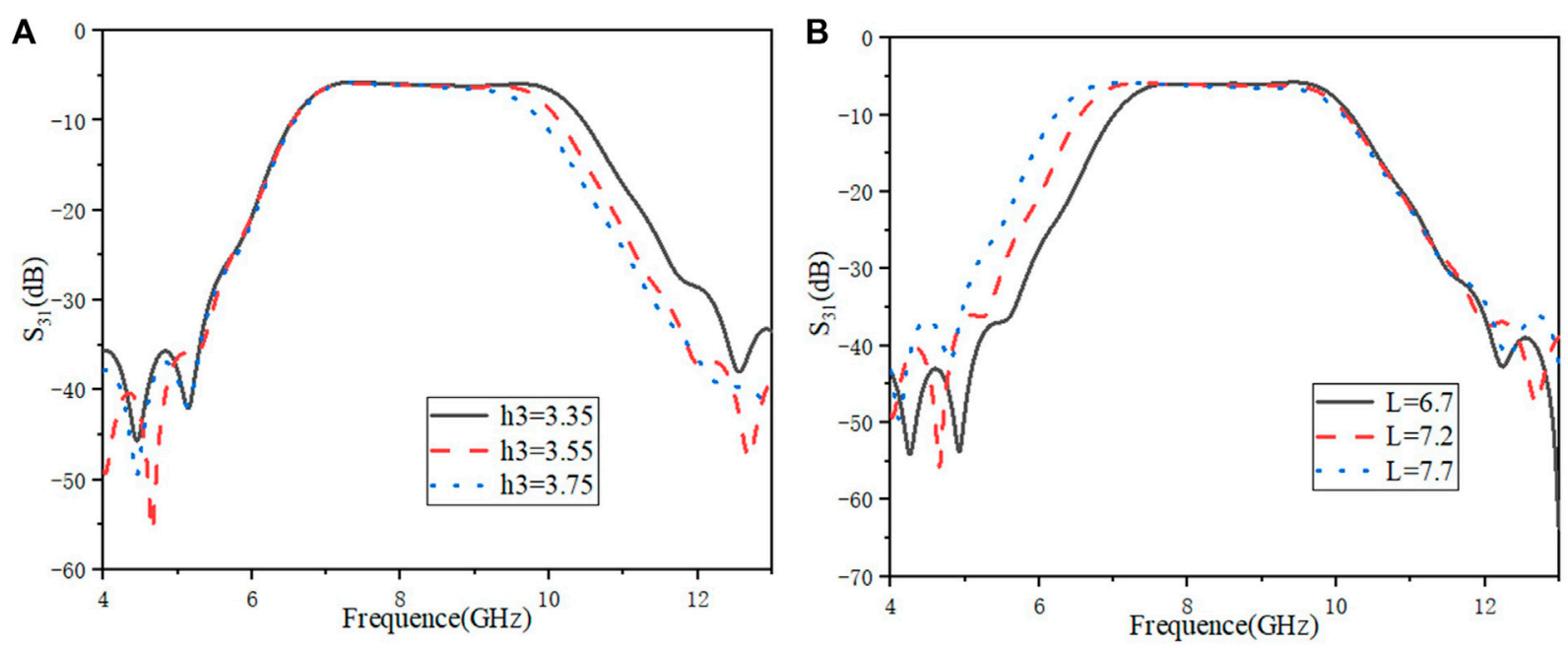

FIGURE 4 | Simulated transmission coefficients $\left(S_{31}\right)$ with different dimensions of (A) h3 and (B) L. 


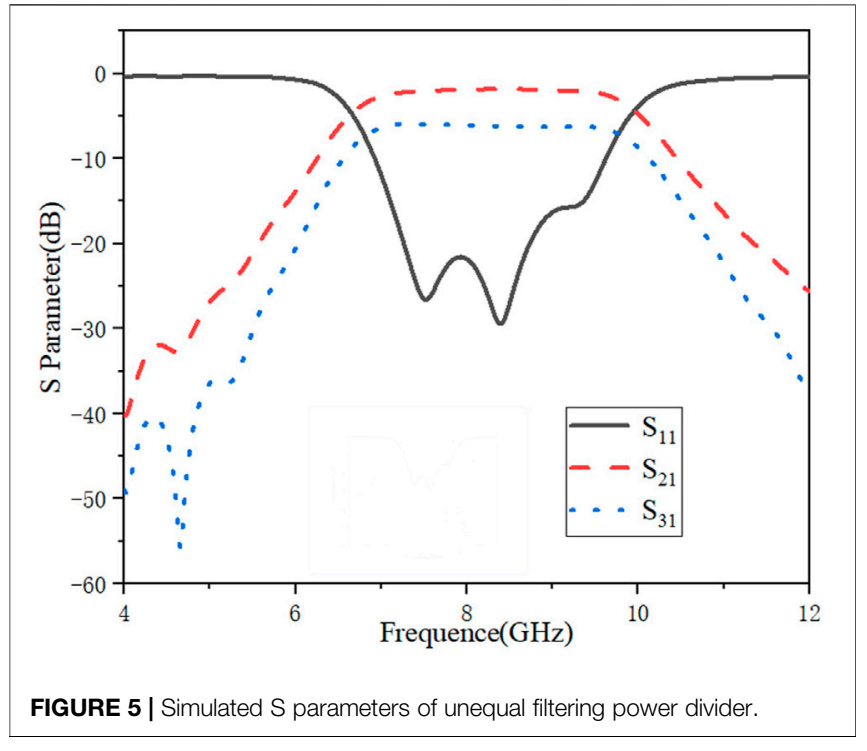

DGSs is introduced. The DGSs are loaded on the bottom layer of the hybrid HMSIW-SSPP units to improve the upper stopband rejection. The structure of the DGS unit is shown in the inset of Figure 6A, in which the red line represents the contour of the grooves on the top layer. The DGS is etched on the bottom layer and it is in the middle of two adjacent grooves. The dimensions of the DGS decide the frequency of its transmission zero. And the optimized dimensions are $\mathrm{a} 1=1.9 \mathrm{~mm}, \mathrm{a} 2=4.4 \mathrm{~mm}, \mathrm{a} 3=3.8 \mathrm{~m}, \mathrm{t}$ $=0.6 \mathrm{~mm}, \mathrm{~s}=0.45 \mathrm{~mm}, \mathrm{~g}=0.25 \mathrm{~mm}$. The comparison of $S_{21}$ of the design with and without the DGSs is shown in Figure 6A. The transmission with two-element array of DGSs provides an extra transmission zero, providing better out-of-band suppression. The cut-off efficiency of transmission zero of DGS at $10 \mathrm{GHz}$ can be improved by increasing the number of DGSs used in the model. A small shift of the cutoff frequency of SSPPs is observed due to the coupling between DGSs and SSPPs. The transmission of the proposed design loaded with DGSs are shown in Figure 6B. An unequal power divider with bandpass filtering effect is observed within band from 6.5 to $9.5 \mathrm{GHz}$. The simulated $S_{11}$
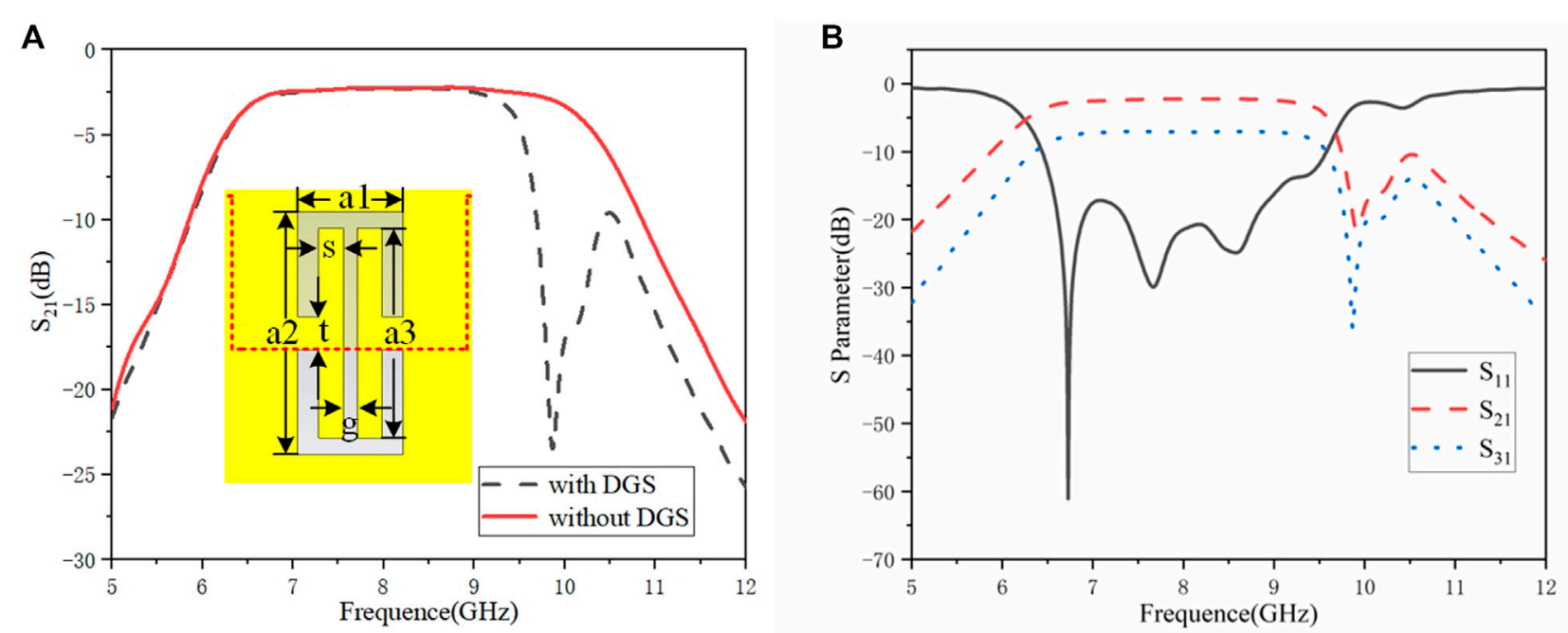

FIGURE 6 | (A) the comparison of $S_{21}$ of the designs with and without DGSs. (B) Simulated S-parameters of the design with DGS.

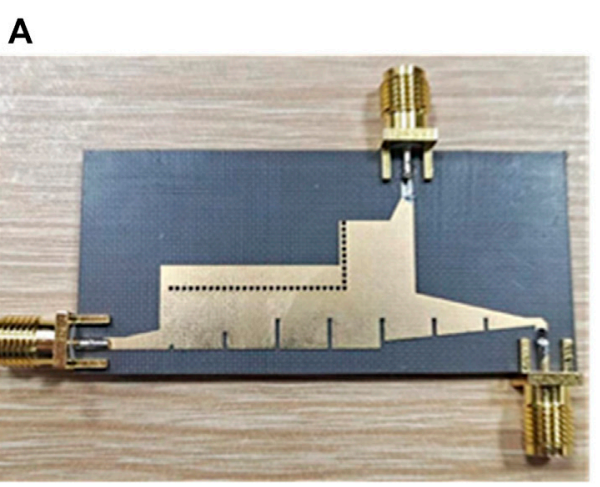

B

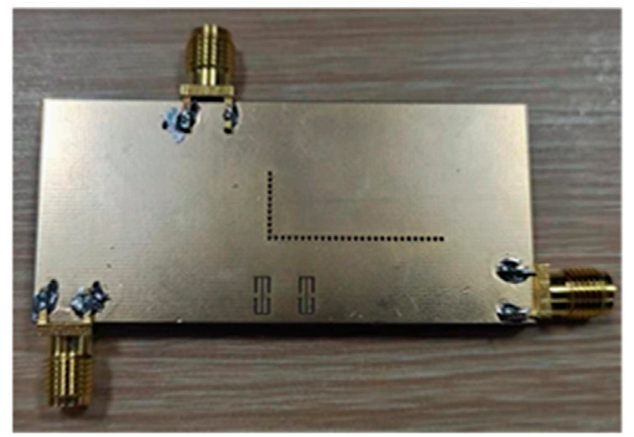

FIGURE 7 | (A) Top view and (B) back view of the prototype. 


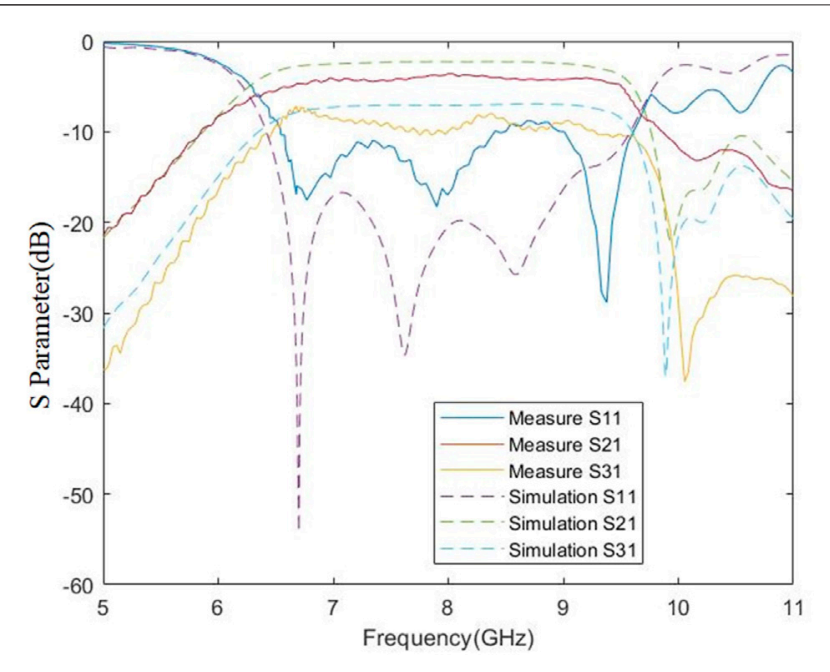

FIGURE 8 | Comparison of simulated and measured S-parameters.

is lower than $-20 \mathrm{~dB}$ in the passband. $S_{21}$ and $S_{31}$ are $-2.2 \mathrm{~dB}$ and $-7 \mathrm{~dB}$, respectively. Within the whole operating band, S21 undulates between $-2.6 \mathrm{~dB}$ and $-2.3 \mathrm{~dB}$, and $\mathrm{S} 31$ undulates between $-7.4 \mathrm{~dB}$ and $-7 \mathrm{~dB}$. The simulations indicate a stable power division. The power division ratio of port 2 and port 3 is $3: 1$.

A prototype of the proposed unequal filtering power divider is fabricated and measured. The substrate is F4B board with relative permittivity of 2.65 and thickness of $1 \mathrm{~mm}$. Photographs of the prototype are shown in Figure 7. The total dimensions of the proposed design are $45 \mathrm{~mm} \times 25 \mathrm{~mm} \times 1 \mathrm{~mm}$. Figure 8 shows the compared of the simulated and measured S-parameters. The measured transmissions are about $1 \mathrm{~dB}$ lower than the simulated ones, because of the machining accuracy of the sample and the unskilled welding of SMA connectors in the

\section{REFERENCES}

1. Deslandes D, Wu K. Integrated Microstrip and Rectangular Waveguide in Planar Form. IEEE Microw Wireless Compon Lett (2001) 11(2):68-70. doi:10. $1109 / 7260.914305$

2. Nedelchev M, Stosić B, Iliev I, Ilieva A, Dončov N. "Wave-based Digital Model of Substrate-IntegratedWaveguide (SIW) Filters for K Band," In 2020 43rd International Conference on Telecommunications and Signal Processing (TSP); Milan, Italy (2020). p. 325-8. doi:10.1109/TSP49548.2020.9163541

3. Shreyus GS, Khatwani H, Shambavi K. "Power Divider with Substrate Integrated Waveguide and CSRR for C Band Application,” In 2017 Innovations in Power and Advanced Computing Technologies (i-PACT); Vellore, India (2017). p. 1-5. doi:10.1109/IPACT.2017.8245019

4. Xu H-X, Wang G-M, Zhang C-X, Yu Z-W, Chen X. Composite Right/leftHanded Transmission Line Based on Complementary Single-Split Ring Resonator Pair and Compact Power Dividers Application Using Fractal Geometry. IET Microw Antennas Propag (2012) 6(9):1017-25. doi:10.1049/ iet-map.2011.0427

5. Moznebi A-R, Afrooz K. Compact Power Divider Based on Half Mode Substrate Integrated Waveguide (HMSIW) with Arbitrary Power Dividing Ratio. Int J Microw Wireless Technol (2017) 9(3):515-21. doi:10.1017/ S1759078716000544 experiments. The operating bandwidth and power division effect keeps steady.

\section{CONCLUSION}

In this paper, an unequal filtering power divider based on hybrid HMSIW-SSPP mode is proposed. The passband can be controlled independently by changing the dimensions of HMSIW and SSPPs. DGSs are introduced on the bottom layer of the device to improve the out-of-band suppression. A prototype working from 6.5 to $9.5 \mathrm{GHz}$ is designed and fabricated. And power division ratio of $1: 3$ is obtained. Such design provides solutions for miniaturized multi-functional devices, and could be used in wireless communication systems.

\section{DATA AVAILABILITY STATEMENT}

The original contributions presented in the study are included in the article/Supplementary Material, further inquiries can be directed to the corresponding author.

\section{AUTHOR CONTRIBUTIONS}

All authors listed have made a substantial, direct, and intellectual contribution to the work and approved it for publication.

\section{FUNDING}

This work is supported partly by Open Research Program of State Key Laboratory of Millimeter Wave under contract K202221, partly by Zhejiang Provincial Natural Science Foundation of China under contract LQ19F010010, and partly by National Natural Science Foundation of China under contract 61901143.

6. Xu H-X, Liang J-G, Wang G-M, Wu F-T, Zhang C-X. Modelling of Composite Right/left-Handed Transmission Line Based on Fractal Geometry with Application to Power Divider. Microwaves, Antennas \& Propagation, IET (2012) 6:1415-21. doi:10.1049/iet-map.2012.0137

7. Pezhman M, Heidari A. Design of Compact SIW-Based Multi-Aperture Coupler for Ku-Band Applications. 2019 27th Iranian Conference on Electrical Engineering (ICEE). 30 April-2 May 2019, Yazd, Iran (IEEE) 1338-41. (2019). doi:10.1109/iraniancee.2019.8786463

8. Hong W, Liu B, Wang Y, Lai Q, Tang H, Yin XX, et al. "Half Mode Substrate Integrated Waveguide: A New Guided Wave Structure for Microwave and Millimeter Wave Application," In 2006 Joint 31st International Conference on Infrared Millimeter Waves and 14th International Conference on Teraherz Electronics; Shanghai, China (2006). p. 219. doi:10.1109/ICIMW.2006.368427

9. Cui Y, Xu K-D, Guo Y-J, Chen Q. Half-mode Substrate Integrated Plasmonic Waveguide for Filter and Diplexer Designs. J Phys D: Appl Phys (2022) 55: 125104. doi:10.1088/1361-6463/ac44bf

10. Ma HF, Shen X, Cheng Q, Jiang WX, Cui TJ. Broadband and HighEfficiency Conversion from Guided Waves to Spoof Surface Plasmon Polaritons. Laser Photon Rev (2014) 8(1):146-51. doi:10.1002/lpor. 201300118

11. Garcia-Vidal FJ, Martín-Moreno L, Pendry JB. Surfaces with Holes in Them: New Plasmonic Metamaterials. J Opt A: Pure Appl Opt (2005) 7(2): S97-S101. doi:10.1088/1464-4258/7/2/013 
12. Jaiswal RK, Pandit N, Pathak NP. Spoof Surface Plasmon Polaritons Based Reconfigurable Band-Pass Filter. IEEE Photon Technol Lett (2019) 31(3): 218-21. doi:10.1109/LPT.2018.288900710.1109/lpt.2018.2889007

13. Xu K-D, Lu S, Guo Y-J, Chen Q. High-order Mode of Spoof Surface Plasmon Polaritons and its Application in Bandpass Filters. IEEE Trans Plasma Sci (2021) 49(1):269-75. doi:10.1109/tps.2020.3043889

14. Wu Y, Li M, Yan G, Deng L, Liu Y, Ghassemlooy Z. Single-conductor Co-planar Quasi-Symmetry Unequal Power Divider Based on Spoof Surface Plasmon Polaritons of bow-tie Cells. AIP Adv (2016) 6(10):105110. doi:10.1063/1.4966051

15. Gao X, Zhou L, Yu XY, Cao WP, Li HO, Ma HF, et al. Ultra-wideband Surface Plasmonic Y-Splitter. Opt Express (2015) 23(18):23270. doi:10.1364/OE.23.023270

16. Gao X, Zhang HC, He PH, Wang ZX, Lu J, Yan RT, et al. Crosstalk Suppression Based on Mode Mismatch between Spoof SPP Transmission Line and Microstrip. IEEE Trans Compon., Packag Manufact Technol (2019) 9(11): 2267-75. doi:10.1109/TCPMT10.1109/tcpmt.2019.2931373

17. Tang X, Zhang Q, Hu S, Chen Y. "Spoof Surface Plasmon Polaritons (SSPP) for Endfire Radiation," In 2018 IEEE Asia-Pacific Conference on Antennas and Propagation (APCAP); Auckland, New Zealand (2018). p. 414-5. doi:10.1109/ APCAP.2018.8538201

18. Zhang Q, Zhang HC, Wu H, Cui TJ. A Hybrid Circuit for Spoof Surface Plasmons and Spatial Waveguide Modes to Reach Controllable Band-Pass Filters. Sci Rep (2015) 5:16531. doi:10.1038/srep16531

19. Guan D-F, You P, Zhang Q, Xiao K Hybrid Spoof Surface Plasmon Polariton and Substrate Integrated Waveguide Transmission Line and its Application in Filter. IEEE Trans Microwave Theor Techn. (2017) 65(12):4925-32. doi:10. 1109/tmtt.2017.2727486

20. Li T, Wang G-M, Lu K, Xu H-X, Liao Z-H, Zong B. Novel Bandpass Filter Based on Csrr Using Koch Fractal CurveIn PIER Lett (2012) 28:121-8. doi:10. 2528/PIERL11082903
21. Pan BC, Luo GQ, Liao Z, Cai JL, Cai BG. Wideband Miniaturized Design of Complementary Spoof Surface Plasmon Polaritons Waveguide Based on Interdigital Structures. Sci Rep (2020) 10:3258. doi:10.1038/s41598-02060244-7

22. Pan BC, Yu P, Liao Z, Zhu F, Luo GQ. A Compact Filtering Power Divider Based on Spoof Surface Plasmon Polaritons and Substrate Integrated Waveguide. IEEE Microw Wireless Compon Lett (2021) 1-4. doi:10.1109/ lmwc.2021.3116169

23. Han Y, Liu Z, Zhang C, Mei C, Chen Q, Hu K, et al. . A Flexible Microstrip Low-Pass Filter Design Using Asymmetric Pi-Shaped DGS. Ieee Access (2019) 7:49999-50006. doi:10.1109/access.2019.2910350

Conflict of Interest: The authors declare that the research was conducted in the absence of any commercial or financial relationships that could be construed as a potential conflict of interest.

Publisher's Note: All claims expressed in this article are solely those of the authors and do not necessarily represent those of their affiliated organizations, or those of the publisher, the editors and the reviewers. Any product that may be evaluated in this article, or claim that may be made by its manufacturer, is not guaranteed or endorsed by the publisher.

Copyright (c) 2022 Pan, Yu, Guo, Qian and Luo. This is an open-access article distributed under the terms of the Creative Commons Attribution License (CC BY). The use, distribution or reproduction in other forums is permitted, provided the original author(s) and the copyright owner(s) are credited and that the original publication in this journal is cited, in accordance with accepted academic practice. No use, distribution or reproduction is permitted which does not comply with these terms. 